\section{Sterbehilfe: die nächsten Schritte}

M. Leuthold

Mit dem Symposium zum Thema "Sterbehilfe» vom Oktober 2000 verfolgte die Schweizerische Akademie der Medizinischen Wissenschaften (SAMW) zwei Ziele: Einerseits wollte sie damit einen Beitrag leisten zur öffentlichen Debatte über die Frage des Sterbens, die erst im Entstehen begriffen und dringend notwendig ist; andererseits sollte das Symposium auch dazu dienen, die eigene Position zu überprüfen und allfälligen Handlungsbedarf festzustellen. Dieses letztgenannte Anliegen stand im Zentrum des zweiten Tages des Symposiums, welcher etwa 40 der Akademie nahestehende Teilnehmerinnen und Teilnehmer versammelte. Einleitend referierte Prof. Gerrit Kimsma, Arzt und Ethiker aus Amsterdam, über die Regelung der Sterbehilfe in den Niederlanden. Anschliessend diskutierten und definierten die Anwesenden die möglichen/wünschbaren kurz- und mittelfristigen Aufgaben der Akademie im Bereich «Sterbehilfe».

\section{Die Situation in den Niederlanden}

Die Debatte im niederländischen Parlament über die gesetzliche Regelung der aktiven Sterbehilfe, welche im Spätherbst 2000 in ganz Europa je nach Standpunkt mit Erstaunen, Bewunderung oder Entsetzen verfolgt wurde, blickt auf eine fast dreissigjährige Entwicklung zurück. Diese Entwicklung war geprägt von einzelnen, wichtigen Gerichtsentscheiden: 1973 hielt ein Gericht fest, dass es keine Pflicht gibt, ein Leben unter allen Umständen zu verlängern; 1984 urteilte der Oberste Gerichtshof, dass der ärztlich assistierte Suizid in gewissen Fällen zulässig sei, und 1994 stellte derselbe Gerichtshof fest, dass auch ein schweres psychisches Leiden ein akzeptabler Grund sein könne, ein Leben zu beenden.

In den Niederlanden wird von einer Strafverfolgung bei ärztlich assistiertem Tod (dieser Begriff umfasst sowohl die direkte aktive Sterbehilfe als auch den ärztlich assistierten Suizid) abgesehen, wenn folgende Bedingungen erfüllt sind:
- Der Wunsch des Patienten muss unbeeinflusst, andauernd und konstant sein.

- Das Leiden muss unheilbar, ohne Hoffnung auf Besserung und langdauernd sein.

- Die Meinung eines zweiten Arztes muss eingeholt werden.

- Es muss ein umfassender detaillierter Bericht erstellt werden.

- Es ist gesetzlich vorgeschrieben, dass jeder unnatürliche Todesfall gemeldet werden muss.

Die niederländischen Definitionen vermeiden die Terminologie von aktiver und passiver, direkter und indirekter Sterbehilfe, weil diese eher als verwirrend denn als klärend empfunden wird. Unterschieden wird zwischen:

- Euthanasia: the ending of someone's life at his or her request;

- PAS: physician assisted suicide;

- NTD: non treatment death;

- APS: death in the course of alleviating pain and suffering;

- LAWER: life ending action without a specific request.

Das niederländische Modell entstand langsam, Schritt für Schritt, und ist das Resultat einer engen $\mathrm{Zu}$ sammenarbeit von Juristen und Medizinern. In der öffentlichen Diskussion konnte so eine Polemik vermieden werden. Die Unterstützung dieses Modells in der Bevölkerung, aber auch bei der Ärzteschaft und dem Pflegepersonal ist dementsprechend gross (rund $90 \%)$.

Seit 1998 sind "Regionale Euthanasie-Evaluations-Komitees» für die Beurteilung der Sterbehilfefälle zuständig; in diesen sind auch Ärzte vertreten. Im ersten Jahr hatten diese 2216 Fälle von Sterbehilfe zu beurteilen; nur einer davon musste an die Strafverfolgungsbehörde weitergeleitet werden. Die Patienten starben in 60\% der Fälle zu Hause; bei 85\% war der Hausarzt involviert. Prof. Kimsma betonte, dass in den Niederlanden für den Umgang mit Sterbenden ein hohes Mass an Fachkompetenzen in den Bereichen Palliativmedizin, Kommunikation und Ethik verlangt wird, und dass die Weiterbildungsangebote entsprechend ausgebaut wurden und werden.

Prof. Kimsma verschwieg die Schwachstellen der aktuellen Lösung nicht: Die Beurteilung des Leidens ist schwierig; oft ist die Beschreibung ungenügend, und mögliche Alternativen werden nicht in Erwägung gezogen. Zudem kommt dem Sterbewunsch des Patienten grosse Bedeutung $\mathrm{zu}$; es ist manchmal schwierig zu bestimmen, ob dieser wirklich unbeeinflusst und konstant ist, und ob der Patient seinen Wunsch bei vollem Bewusstsein äussert. Ebenso ist unklar, wann der beste Zeitpunkt für das Einholen einer Zweitmeinung ist und wie deren Unabhängigkeit sichergestellt werden kann. Prof. Kimsma gab zusammenfassend seiner Überzeugung Ausdruck, dass nicht nur die Hilfe beim Sterben, sondern auch die Hilfe zum Sterben dem ärztlichen Ethos entspricht.
Korrespondenz:

Dr. Margrit Leuthold

SAMW

Petersplatz 13

CH-4051 Basel

E-mail: leuthold@samw.ch 


\section{Erwartungen an die SAMW}

Im Anschluss an das Referat von Prof. Gerrit Kimsma zogen sich die Teilnehmer und Teilnehmerinnen zu zwei getrennten Workshops zurück. Unter dem Oberthema "Handlungsbedarf für die SAMW?» befasste sich die eine Gruppe mit den Erfahrungen einer liberalen Praxis in anderen Ländern und den möglichen Konsequenzen einer Liberalisierung für Ärzteschaft, Pflege und Patienten in der Schweiz; die andere Gruppe hatte den Auftrag, Forschungsprojekte zur Erarbeitung von Entscheidungsgrundlagen zu skizzieren sowie einen Massnahmenkatalog zur Förderung der Palliativmedizin und -pflege aufzustellen. Die Tatsache, dass beide Gruppen nach anderthalb Stunden Diskussion ähnliche Resultate präsentierten, löste im ersten Moment eine gewisse Verblüffung aus, wurde dann aber von den SAMW-Verantwortlichen als Zeichen dafür genommen, dass die verschiedenen Bereiche einerseits nicht klar voneinander abzugrenzen sind, und dass andererseits die vorgeschlagenen Massnahmen naheliegend und realistisch sind.

\section{Datengrundlagen}

Wer immer im Laufe des Symposiums Zahlen zur Sterbehilfe zitieren wollte, musste auf ausländisches Datenmaterial zurückgreifen. In der Schweiz gibt es noch keine Studien, welche - neben der eigentlichen Todesursache - auch die Art und Weise und den Ort des Sterbens erfassen. Ebenso gibt es keine Angaben darüber, was die Ärzteschaft und das Pflegepersonal von der Sterbehilfe denken, und nur unvollständige Angaben über die diesbezügliche Einstellung der Bevölkerung. Konkret wurden folgende Fragestellungen genannt, die bearbeitet werden könnten: Häufigkeit verschiedener Praktiken der Sterbehilfe/Sterbebegleitung/Palliativtherapie? Gibt es neben den bekannten, in der Schweiz erlaubten Praktiken der Ärzteschaft auch zusätzliches, bisher nicht thematisiertes Handeln gegenüber todkranken Patienten? Der «Beihilfe zum Suizid", welche in der Schweiz nicht strafbar ist, sollte besondere Beachtung geschenkt werden (auch wenn sie von den SAMW-Richtlinien nicht als ärztliches Handeln bezeichnet wird), und zwar in Form sowohl von retrospektiven als auch prospektiven Studien. In Zürich sind am Institut für Rechtsmedizin und am Institut für Sozial- und Präventivmedizin Studien geplant, welche die beschriebenen Lücken schliessen sollen. Es ist zu prüfen, ob dadurch die SAMW auf das Lancieren eigener Projekte verzichten und stattdessen diese anlaufenden Studien unterstützen soll.

\section{Palliativmedizin}

Es besteht Einigkeit darüber, dass palliativ-medizinische Massnahmen, richtig eingesetzt, die Lebensqualität Schwerstkranker und Sterbender ausserordentlich erhöhen. Sie verändern deshalb auch den Wunsch nach Sterbehilfe. Die Palliativmedizin und deren Möglichkeiten sind in der Schweiz jedoch zuwenig bekannt. Das Angebot ist minimal und geprägt durch die persönliche Initiative weniger Einzelpersonen; an offizieller Unterstützung und Förderung mangelt es hingegen erheblich. In öffentlichen Spitälern existieren nur gerade drei Stationen, dazu eine Station in einer Privatklinik. Ausserdem gibt es vier Hospize und zwei Konsiliardienste. Da es Anzeichen gibt, dass in einem solchen von Palliativpflege geprägten Umfeld Sterbewünsche Schwerstkranker seltener auftreten, sind die Propagierung und Förderung der Palliativmedizin eine der Hauptforderungen der beiden Gruppen. Die SAMW sollte sich - zusammen mit anderen Organisationen - dafür einsetzen, dass jedem Menschen am Ende seines Lebens der Zugang zu palliativer Pflege ermöglicht wird. Dazu gehören eine massive Ausweitung des Angebotes sowie - als Vorbedingung - entsprechende Aus- und Weiterbildungsangebote. Die SAMW sollte ausserdem darauf hinwirken, dass in der Ärzteschaft dem Konzept "Verbesserung der Lebensqualität vor Lebensverlängerung» mehr Gewicht zukommt.

\section{Aus-, Weiter- und Fortbildung}

Wie die Erfahrungen in den Niederlanden zeigen, verlangt der Umgang mit Sterbenden neben Kompetenzen im Bereich Palliativmedizin auch ein hohes Mass an ethischer und kommunikativer Kompetenz. In beiden Hinsichten stellten die Anwesenden beträchtliche Defizite in der ärztlichen Praxis fest. Es ist daher in der ärztlichen Aus-, Weiter- und Fortbildung darauf zu achten, dass diese Kompetenzen geschult werden. Für die Curricula sind dementsprechend neue Konzepte zu entwickeln.

Die Haltung und das Handeln vis-à-vis von Sterbenden und dem Sterben sind zentrale Aspekte des Arztseins; das Bewusstsein dafür zu fördern und auf die Aufnahme solcher Inhalte in die Curricula hinzuwirken, sahen die Teilnehmerinnen und Teilnehmer als wichtige Aufgabe der SAMW an. Die SAMW könnte zudem ihr Netzwerk nutzen, um den Fachgesellschaften geeignete Expertinnen und Experten für Fortbildungsveranstaltungen zu vermitteln.

\section{Richtlinien}

Seit über dreissig Jahren erarbeitet die SAMW Richtlinien oder Empfehlungen zu Bereichen, die von der Öffentlichkeit oder der Ärzteschaft als heikel angesehen werden. Sie besitzen zwar keine Gesetzeskraft, sie geniessen aber hohe moralische Anerkennung. Es wird erwartet, dass die SAMW ihre medizinisch-ethischen Richtlinien «Betreuung Sterbender und zerebral schwerstgeschädigter Patienten", welche letztmals im Jahre 1995 überarbeitet wurden, kritisch überprüft und neue Erkenntnisse mit berücksichtigt. Ebenso, dass sie für den Bereich "Palliativmedizin» die Erarbeitung von Empfehlungen ins Auge fasst, um so auf die Bedeutung dieser Disziplin hinzuweisen. 


\section{Erste Schritte sind eingeleitet}

Das Thema "Sterbehilfe» bleibt in der nächsten Zeit auf der politischen Agenda. So wird das Parlament in naher Zukunft die Antwort des Bundesrates auf den "Bericht Sterbehilfe» diskutieren; ebenso wird es sich mit einem entsprechenden Vorstoss von Nationalrat Franco Cavalli befassen müssen.

Die SAMW wird versuchen, sich in der anstehenden Debatte weiterhin Gehör zu verschaffen. Bereits hat sie auch begonnen, die ihr im Rahmen des Symposiums "Sterbehilfe» zugedachten Aufgaben anzu- gehen: Eine Subkommission der Zentralen Ethikkommission hat den Auftrag erhalten, die Richtlinien zur Sterbehilfe zu überarbeiten; das Generalsekretariat hat erste Kontakte mit der Schweizerischen Gesellschaft für Palliativmedizin geknüpft; und Mitte Februar hat der Vorstand beschlossen, die Studie «Medical End-of-Life Decisions: Attitudes and Practices in 6 European Countries» (in der Schweiz durchgeführt durch das ISPM Zürich) nicht nur ideell, sondern auch mit einem namhaften finanziellen Beitrag zu unterstützen. 